\title{
e-PARFIT: An Extended Partition-Based Approach in Automated Fingerprint Identification System for Partial Images
}

\author{
${ }^{*}$ Ankit Shrivastava, ${ }^{2}$ Devesh Kumar Srivastava \\ ${ }^{1,2}$ School of Computing and Information Technology, Manipal University Jaipur, Rajasthan, India-303007 \\ Email: ankitshrivastavaieee@gmail.com,devesh988@yahoo.com
}

\section{Received: 09 ${ }^{\text {th }}$ July 2018, Accepted: $14^{\text {th }}$ August 2018, Published: $31^{\text {st }}$ August 2018}

\begin{abstract}
It is the intention of this work to develop a system which specialized in processing scanned images. This paper focuses on developing an extended partitioning approach for partial fingerprint identification. The procedure has utilized image orientation, enhancement, extended-partitioning, binarization and feature extraction. The major focus of this work is to reduce error rates during match. The proposed approach performs partitioning on images to create extended sub-parts before matching. This procedure clinically extracts the information of sub-parts and establishes a lower bound on the performance. The extended partition-based representation combines both the global and the local information present in a fingerprint. Test results have presented that the proposed method has a discriminatory power compared to other representations. Finally, it has shown that the use of all the techniques presented in this work significantly improves error rates of a partial fingerprint identification system on a large database.
\end{abstract}

Keywords: Partial Fingerprint Image, Image Orientation, Image Enhancement, Image Thinning, Extended Partitioning, Binarization, Feature Extraction.

\section{Introduction}

Fingerprints have been experimentally considered for various years in our general public. The attributes of fingerprints were examined as right on time as the 1600s. In the vicinity of 1896 and 1897, Sir Edward Henry built up the Henry Classification Framework, which rapidly discovered overall acknowledgment inside a couple of years. This framework takes into account legitimate order of a total arrangement of the ten finger-print images for a man. By setting up groupings in view of unique finger-prints design sorts, the Henry Framework enormously diminishes the exertion of looking through a huge database. [1]

As finger-prints were used in more fields, the quantity of solicitations for unique finger-print coordinating started to increment consistently. In the meantime, the span of the databases kept on extending with each passing day. In this manner, it soon ended up noticeably troublesome for groups of finger-print specialists to give precise outcomes in a convenient way. These frameworks took into account a change in operational profitability among law requirement organizations. In the meantime, the computerized frameworks lessened subsidizing necessities to contract and prepare human finger-print specialists. Today, automated finger-print identification system can be found in an extensive variety of nonmilitary personnel applications. [2]

Keeping in mind the end goal to a human physiological or behavioural trademarks to be utilized as a gainful biometric quality, it must fulfil four criteria. Most importantly, it should be universal, with every individual possessing the given trademark. Furthermore, it ought to be a particular quality, which means there is a noteworthy difference in the trademark between any two given people. Next, there should be a sure perpetual quality intrinsic to the component, i.e., the deliberate components ought to remain moderately constant over some stretch of time. Lastly, the credit ought to be anything but difficult to gather and measured quantitatively. Different issues, for example, execution, agreeableness, and circumvention, should be inspected for a framework that actualizes individual acknowledgment with biometrics [3].

\section{Background}

A finger-print design is involved an arrangement of edges and valleys. In a finger-print image, the edges show up as dark lines while the valleys are the light regions between the edges. A slice or consume to a finger does not influence the fundamental edge structure, and the first example will be replicated when new skin develops. Edges and valleys for the most part run parallel to each other, and their examples can be investigated on a worldwide and nearby level. At the worldwide level, the finger-print image will have at least one areas where the edge lines have a particular shape. These shapes are normally portrayed by zones of high ebb and flow or continuous edge endings and are known as particular region. The three essential sorts of these particular region are loop, delta, and whorl, cases of which are appeared in Figure 1.

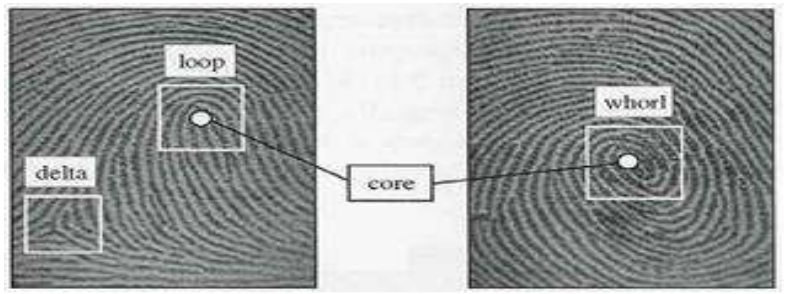

Fig. 1.Singular Regions and Core Points

Many coordinating calculations utilize the focal point of the most astounding loop sort peculiarity, known as the centre, to pre-adjust finger-print images for better outcomes. As appeared in Figure 2, these three essential singularities help shape the five noteworthy classes of fingerprints [4]. 


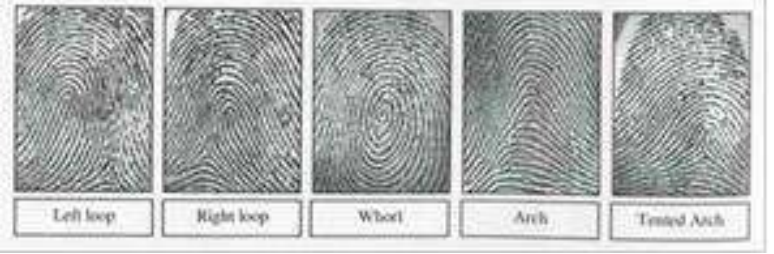

Fig. 2.Examples of Fingerprint Classes

While the worldwide level takes into consideration a general order of fingerprints, examining the picture at the neighbourhood level gives a lot of detail. These subtle elements are gotten by watching the areas where an edge ends up plainly irregular, known as minutiae point. The most widely recognized sorts of features are appeared in Figure 3. As a rule, an edge can either arrive at an end, which is known as an end, or it can part into two edges, which is known as a bifurcation. Alternate sorts of particulars are somewhat more muddled blends of terminations and bifurcations. For instance, a lake is essentially a grouping of two bifurcations in contradicting headings, while an autonomous edge highlights two separate terminations inside a nearby separation. The FBI particulars arrange show considers just terminations and bifurcations inside a unique mark picture. On the whole, investigating a unique mark on the nearby level gives the important data to precisely recognize one finger impression from another.

\begin{tabular}{|l|l|}
\hline & Termination \\
\hline & Bifurcation \\
\hline & Lake \\
\hline & Independent ridgse \\
\hline & Point or istand \\
\hline & Spar \\
\hline
\end{tabular}

Fig. 3. Basic Types of Minutiae

\section{Related work}

Numerous programmed fingerprint recognition methodologies have been proposed. Among the different fingerprint portrayal strategies, the details based fingerprint portrayal and coordinating are generally utilized by both machine and human specialists. Details portrayal has a few inconveniences regarding the format size and its discriminability. In this work, we will concentrate on partition based frameworks. Different methodologies that dodge arrangement and match nearby highlights have been additionally proposed. Nonetheless, there has been no examination that expressly tends to the issues of halfway fingerprint coordinating. The investigation on the security quality of a fingerprint acknowledgment framework contrasted with a watchword framework has been led as of late. They make suspicions that the two taking an interest fingerprints have a similar number of highlight focuses and have a fixed picture measure. The security quality of a fingerprint framework lessens when the span of fingerprint diminishes. Be that as it may, the connection between fingerprint measure and the framework security quality and how to keep up the security level of halfway fingerprint acknowledgment has not been tended to. Highlight extraction calculations exhibited in writing can be extensively classified as those in view of direct extraction from dark scale pictures and those in view of binarization. [5]

Image-based approach

Image based systems are another huge classification of unique mark coordinating. These procedures are engaging on the grounds that they don't require a lot of pre-handling to create worthy outcomes. Much of the time, the main pre-handling strategies that are connected are a binarization and diminishing stage. Along these lines, imaged-based strategies have a superior computational effectiveness than the standard particulars based methods. Additionally, for low quality finger-print images, image based strategies create preferred outcomes over particulars extraction techniques, where it might be hard to dependably remove the genuine details focuses [6].

Skeleton based approach

A critical segment for image coordinating is managing pivot. Since the information picture may be situated uniquely in contrast to the layout picture, it is important to apply a rotational rectification to accomplish the best outcomes. Numerous frameworks superimpose the info picture with the format picture and register the relationship between comparing pixel esteems for an assortment of relocation and rotational esteems. The greatest relationship esteem delivered in this procedure identifies with the most ideal arrangement between the info and the layout [7].

Wavelet-based approach

Another strategy for finger-print identification framework includes wavelets, where finger-prints designs are coordinated in view of wavelet area highlights. An essential preferred standpoint to this approach is that these highlights can be straightforwardly extricated from the unique mark picture without applying any pre-preparing steps. Once the center point has been resolved, a rectangular area encompassing the center is set up, which is alluded to as the focal sub-picture. This territory is then isolated into non-covering square pieces of uniform size. From here, the wavelet disintegration is figured on each piece, and its wavelet highlights are computed. Next, a worldwide component vector is shaped, which incorporates the highlights separated from each square of the focal sub-picture. Once the element extraction has been performed, it is conceivable to direct a coordinating succession with the format highlights. The lower computational prerequisites of this procedure make utilizing wavelet highlights alluring to a little scale framework. [8] 
Ridge feature-based approach

Breaking down different edge highlights gives this flexibility. There are a few highlights that are regularly analysed in the present frameworks, running from genuinely essential to more progress. At the essential end, the physical size and state of the outer unique mark outline can be registered. This approach offers a few information when little else can be removed. On a marginally further developed level, the spatial relationship and geometrical characteristics of the edge lines can be inspected. Additionally, gathering worldwide and nearby surface data is another alternative. A last edge include that can be dissected is the area of sweat pores inside the edges. Despite the fact that sweat pores are profoundly discriminant among the populace, distinguishing them requires a propelled gathering framework, and their essence would in all likelihood be unnoticeable in low quality pictures. The essential edge highlights, be that as it may, are realistic from any quality picture. Since particulars based strategies require a picture of good quality, edge highlights offer an option for poor pictures. Besides, edge highlight based strategies don't need to be restricted to pictures of low quality. Rather, they can be utilized as a part of conjunction with particulars based methods for pictures of good quality. With more information to be utilized as a part of the coordinating procedure, the precision and vigor of a framework would without a doubt increment. Correlation-based approach

Two unique finger impression pictures are overlaid and the linking between involving pixels is figured for various measures (e.g. different rearrangements and replacements).In request to manage a portion of the issues of the details based approach, they have picked an option approach. Rather than just utilizing the details areas, our technique straightforwardly utilizes the dark level data from the unique mark picture, since a dim level unique finger impression picture recolors substantially wealthier, more biased, data than just the particulars areas. Those areas just portray a little piece of the neighborhood edge valley structures.

The connection based unique finger impression confirmation framework is motivated by remove between the distinctions of lines. It chooses trademark formats in the essential unique mark. At that point, layout coordinating is utilized to end the positions in the auxiliary unique mark at which the formats coordinate best. At last, the layout positions in the two fingerprints are contrasted all together with settle on the choice whether the prints coordinate. [10]

\section{Template adaptation-based approach}

Example based calculations look at the fundamental unique finger impression designs between a formerly put away format and an applicant finger impression. This requires the pictures be adjusted in a similar introduction. To do this, the calculation finds an essential issue in the unique finger impression picture and focuses on that. In an example based calculation, the layout contains the sort, size, and introduction of examples inside the adjusted unique mark picture. The competitor unique mark picture is graphically contrasted with the layout with decide how much they coordinate. The approach, which utilizes picture based techniques, tries to do coordinating in view of the worldwide highlights of an entire unique mark picture. It is a progressed and recently rising technique for unique mark acknowledgment. What's more, it is helpful to tackle some recalcitrant issues of the primary approach. In picture based coordinating, the picture itself is utilized as the layout. It requires just low determination pictures. Coordinating is finished by optical connection and is greatly quick. It depends on the worldwide highlights of an entire unique mark picture. Be that as it may it requires precise arrangement of the unique mark tests and isn't good for changes in scale, introduction and position. [11]

\section{Minutiae-based approach}

This strategy removes details from the unique finger impression pictures, and afterward thinks about this information to the already put away layout informational collections. By and large, the particulars subtle elements are put away as groups of focuses for the two dimensional level. For every minutia, the $\mathrm{x}$-and $\mathrm{y}$ organizes demonstrating its area inside the picture are recorded. Other put away parameters may incorporate the introduction edge of each details and the particular sort of details found. For the most part, details based strategies require a lot of pre-preparing to deliver precise outcomes [12].

Another strategy includes diminishing the unique finger impression picture, at that point playing out a sweep with a three pixel-by-three pixel hinder over the whole picture. This procedure will be clarified in full detail in the up and coming Parts. Another technique fuses a bank of channels to separate the particulars. In particular, the district of premium gets separated in eight unique ways, which totally catches the nearby edge attributes utilizing a bank of Gabor channels. At the point when the Gabor channels are appropriately tuned, they can evacuate clamor while keeping up the genuine edge and valley structures. Since a details point can be viewed as an oddity among locally parallel edges, these focuses can be identified subsequent to applying the bank of Gabor channels. The principle downside of the particulars based approach is the mistake spread from the details extraction to the choice stage. All in all, the separated particulars layouts contain various false details, while additionally some particulars will be remembered fondly. This is particularly the situation when utilizing terrible quality fingerprints. The heuristics don't get all spurious particulars, while they may dismiss a portion of the honest to goodness details. Accordingly, the choice stage needs to look at two influenced sets. [13]

Minuiae-based framework

Minutia extraction (figure 4) incorporates image enhancement, picture division and last extraction forms while particulars coordinating incorporate details arrangement and match forms. Under picture upgrade step histogram adjustment, quick Fourier change builds the nature of the information picture and picture binarization changes over the dark scale picture to a 
parallel picture. At that point picture division is performed which removes a locale of enthusiasm utilizing edge stream estimation. [14]

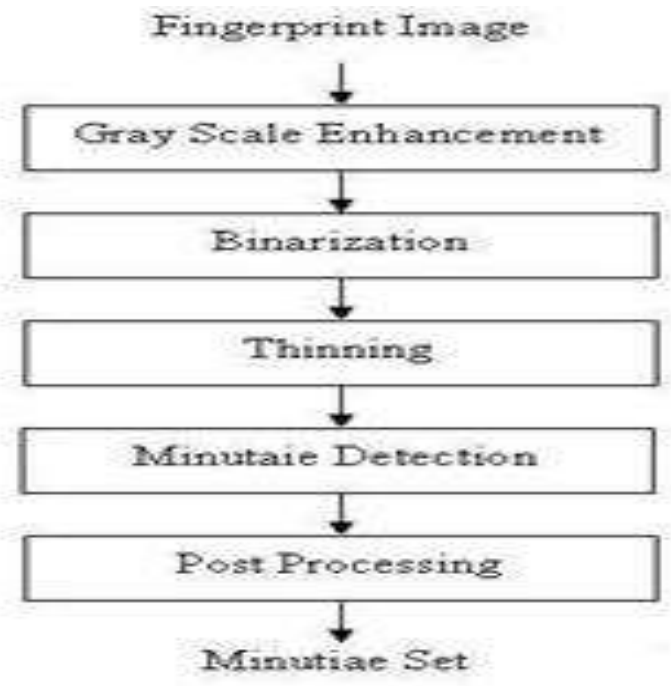

Fig. 4 Minutiae Based Framework

From there on the minutia focuses are separated in the Last Extraction venture by Edge Diminishing, Minutia Stamping and Expulsion of False Particulars forms. Utilizing the above Minutia Extraction process we get the Particulars sets for the two fingerprints to be coordinated. Details Coordinating procedure iteratively picks any two particulars as a kind of perspective minutia combine and after that matches their related edges first. On the off chance that the edges coordinate well, two unique mark pictures are adjusted and coordinating is led for all outstanding minutia to produce a Match Score. Most present day unique finger impression coordinating advancements utilize particulars coordinating.

The thought being whether you can discover enough details in a single picture that have comparing particulars in another picture then the pictures are in all likelihood from a similar unique mark. Details are generally coordinated together by their separation in respect to other particulars around it. In the event that different focuses in one picture have comparative separations.

Issues with minutiae based system

$\square$ The versatility of human skin and dampness can cause twists.

$\square$ To manage fractional or inadequate fingerprints are inclined to disappointment.

$\square$ Security Issues of Partial Fingerprint Matching

$\square$ Minutia-based representation contain only local information.

\section{Proposed system}

The extended partition based finger-print identification approach is fit for managing low quality pictures from which no features can be separated dependably and with fingerprints that experience the ill effects of non-uniform shape contortions. Trials have demonstrated that the execution of our framework is practically identical to the execution of Range Based, Bearing Based, Half and half
Combination Based and Altered Slope Based unique finger impression Distinguishing proof frameworks. Our proposed system is shown in figure 5 . In request to encourage coordinating, the crude computerized unique mark is pre-processed by picture upgrade and picture diminishing, at that point produced improved dark picture is split in 5 pushes and in addition 5 segments, after binarization of parceled picture we get advanced picture which experiences an element extractor to create a smaller however expressive portrayal, called a highlighted esteem. The subsequent portrayal is encouraged to the element matcher, which thinks about it against the all put away format from the framework database.

\section{Image Enhancement}

Customarily, in law requirement applications fingerprints were gained online by exchanging the inked impact on a paper. These days, the computerized fingerprint verification frameworks utilize live-filter advanced pictures of fingerprints procured from a fingerprint sensor.

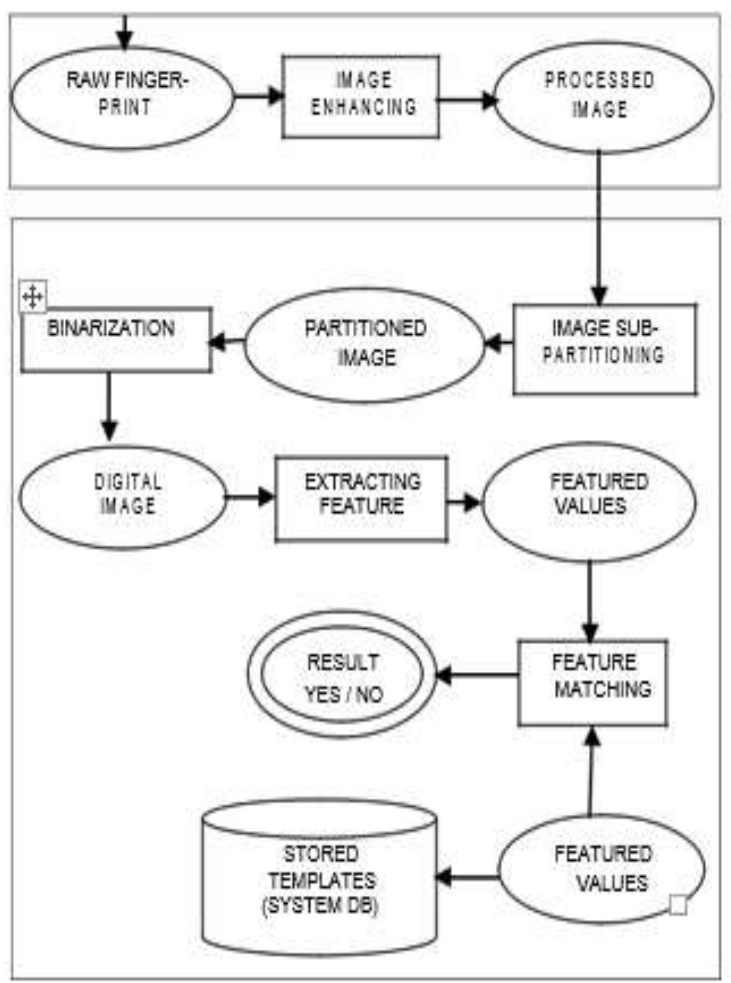

Fig. 5 Proposed System

Various strategies are utilized to gain fingerprints. Out of them, the inked image technique remains well-known one. Inkless unique finger impression scanners are likewise present wiping out the halfway digitization process. Unique mark quality is imperative since it influences specifically the element extraction calculation. Two sorts of corruption more often than not influence unique finger impression pictures: 1) the edge lines are not entirely consistent since they now and then incorporate little breaks (holes); 2) parallel edge lines are not generally all around isolated because of the nearness of jumbling commotion. [15] 
These two issues could be tackled by picture prehandling appeared in figure 6; in which first the crude picture gets upgraded through picture improvement device and second the improved picture nourished to the picture diminishing apparatus to deliver a diminished picture with containing just valuable data. Presently this diminished picture could be utilized for extricating highlights by our segment based approach.

Image enhancement and orientation

A standout amongst the most generally referred to finger-print upgrade strategies is the technique utilized by Hong, which depends on the density of image with Gabor filters applied to region edge introduction and edge recurrence.

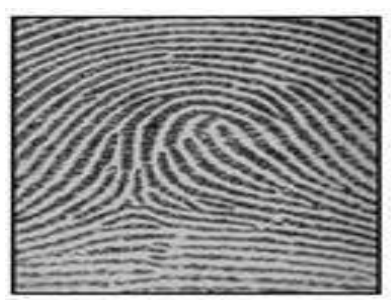

(a)

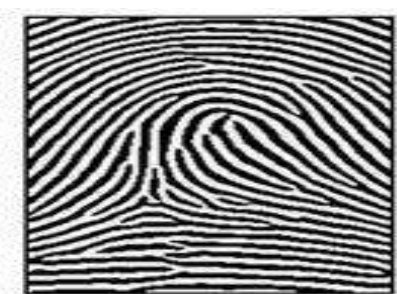

(b)
Fig. 6a) A Raw Fingerprint b) Image after Enhancement

The fundamental phases of this calculation incorporate standardization, edge introduction estimation, edge recurrence estimation and filtering. Unique mark improvement techniques in light of the Gabor filter is broadly used to encourage different applications of fingerprint biometric. [16] An option way to deal with upgrading the highlights in a fingerprint picture is the method utilized by Rajkumar called directional Fourier filtering. The past approach was a spatial space strategy that includes spatial convolution of the picture with filters, which can be computationally costly. On the other hand, working in the recurrence area enables one to efficiently convolve the fingerprint picture with filters of full picture estimate. Locales are characterized by the unique mark edges that bound them. Be that as it may, on account of the idea of the unique mark and current filtering advancements edge detail can miss from the examined unique mark. Over the time of task we will apply an effectively created edge improvement procedure, which will get a portion of the straightforward holes, and white the undesired and futile hole between the edges. [17]. on the off chance that the line is solid then it is no doubt that the edge should traverse the hole and thusly can be attracted. Endpoints are simply pixels that exclusive make them neighbor pixel.

Image thinning

An imperative way to deal with speaking to the basic state of a plane area is to lessen it to a diagram. This lessening might be refined by getting the skeleton of the district by means of diminishing (additionally called skeletonizing) calculation.

Remove end points.

Break connectedness

Image

Acquisition

\section{Edge}

Detection

Thinning Feature

Extractor

To apply diminishing we will utilize the improved Zhang-Suen algorithm[18], A 3x3 window as in figure7; move down all through the picture and estimations Cellwise are completed on each pixel.

\begin{tabular}{|l|l|l|}
\hline$P_{0}$ & $P_{2}$ & $P_{3}$ \\
$(j-1, j-1)$ & $(j-1, j)$ & $(j-1, j+1)$ \\
\hline$P_{1}$ & $P_{1}$ & $P_{4}$ \\
$(j, j-1)$ & $(i, j)$ & $(i, j+1)$ \\
\hline$P_{7}$ & $P_{i}$ & $P_{3}$ \\
$(j+1, j-1)$ & $(j+1, j)$ & $(j+1, j+1)$ \\
\hline
\end{tabular}

Fig. 7 Image Thinning Window [18]

This procedures adequately lighten the picture, in any case, it now and then makes unfortunately ancient rarities.

\section{Extended partitioning}

Extended partitioning implies isolate into sub-parts. Division of an image is a procedure in which any image is divided into parts in a way to extract data. Picture parcelling is a vital handling advance for our calculation utilized for low level element detection assignment. In an acknowledgment sort of articles that may show up in a picture may be separated into pecking order of planes of data or highlights that framework needs to extricate from a picture. Henceforth techniques to remove highlights move toward becoming important. We propose a picture dividing system to deliver an area based picture portrayal. The proposed technique segments the picture into number of lines, segments and cells independently as in figure 8, figure 9 and figure 10 .

Row-wise

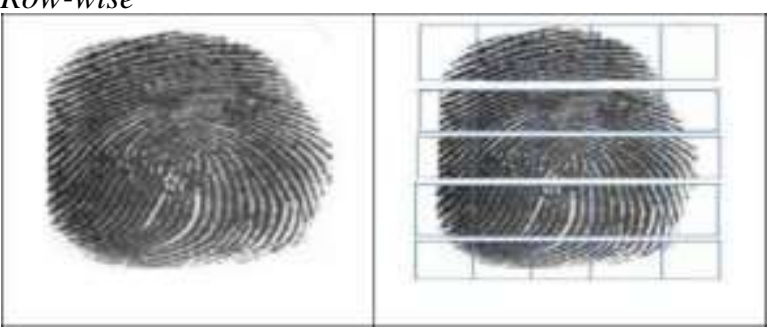

Fig. 8 Extended Partitioning; Row-wise Column-wise

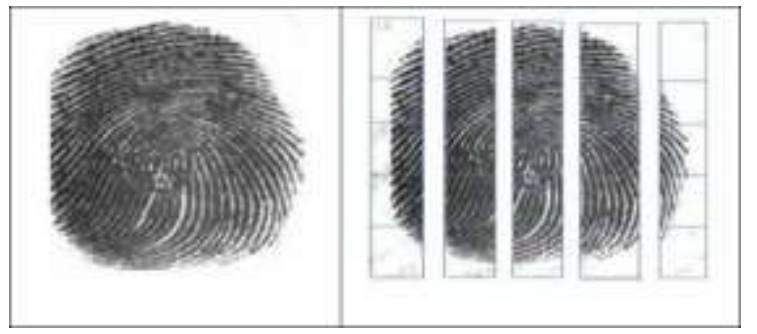

Fig. 9 Extended Partitioning; Column-wise 


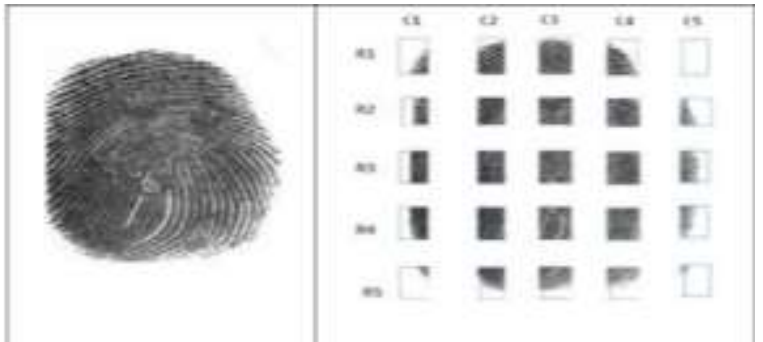

Fig. 10 Extended Partitioning; Cell wise

Those all bits at that point go through the binarization step. We trust that the picture dividing worldview is generally relevant to progressing execution in abnormal state picture understanding errands.

\section{Binarization}

Binarization is an approach by which, the dim scale picture is changed over into a parallel picture before highlight extraction. While calculations vary in a few usage viewpoints, they have the accompanying normal stages. In calculations where diminishing is absent, details recognition is done by means of format coordinating. The details extraction process brings about two types of blunders. The identification may present spurious details where they don't exist in the first or may miss real particulars.

In any case, these systems likewise bomb in low quality pictures. The most proficient methodologies depend on the structure of the unique mark edge structure to play out the division and binarization process. Another binarization method in light of the utilization of edges separated. The subsequent edge picture is utilized as a part of conjunction with the first dim scale picture to get the binarized picture. This depends on the recursive approach of line following and line diminishing. Two versatile windows, the edge window and the dark scale window are utilized as a part of each progression of the recursive procedure. In any case, the pixel with the most reduced dark scale esteem is picked and a window is focused on it. The limit of the window is then inspected to decide the following position of the window. The window is progressively position to follow the edge limit and the recursive procedure ends when all the edge pixels have been taken after to their individual closures. A versatile stream introduction based division or binarization calculation. In this approach the introduction field is registered to get the edge bearings at each point in the picture. To section the edges, a $16 \times 16$ window arranged along the edge heading is considered around every pixel. The projection whole along the edge course is registered. The focuses of the edges show up as pinnacle focuses in the projection. The edge skeleton subsequently acquired is smoothened by morphological operation. At last details are identified by finding the end focuses and bifurcations in the diminished twofold picture. For binarization we enlarge the image until we get $8 \times 8$ size matrix so that each and every cell contains a single pixel value.

Image of any format is converted into ".pgm" format .pgm stands for portable gray map. When Pixel Value lies between -128 to -1 then we consider it as zero and
Pixel Value lies between 0 to 127 then we consider it as one.

\begin{tabular}{|c|c|}
\hline PIXEL VALUE ( PV ) & BINARY-BIT \\
\hline$-128<=\mathrm{PV}<=-1$ & 0 \\
\hline $0<\mathrm{PV}<=127$ & 1 \\
\hline
\end{tabular}

Since in Gray format, pixels are from 0 to 256, so in.pgm format, after binarisation, bits for the pixel values are assigned according to the above conventions.

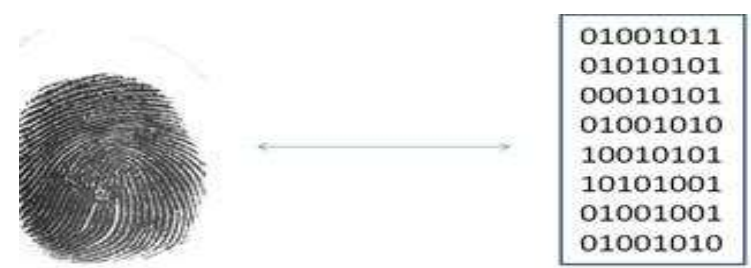

Fig. 11 Concept of Binarization

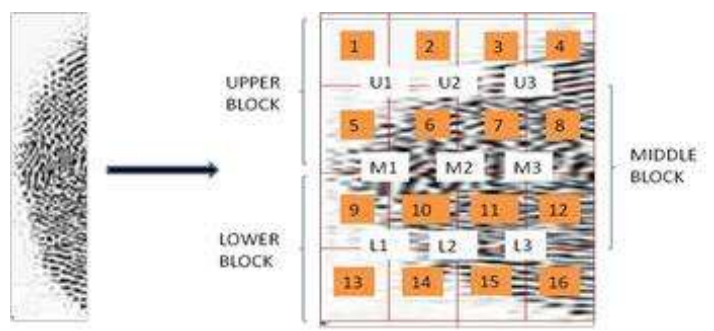

Fig. 12 Binarization Phase - I
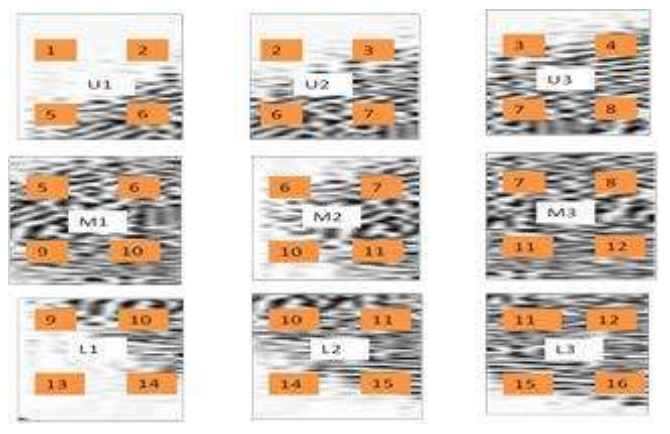

Fig. 13 Binarization Phase - II

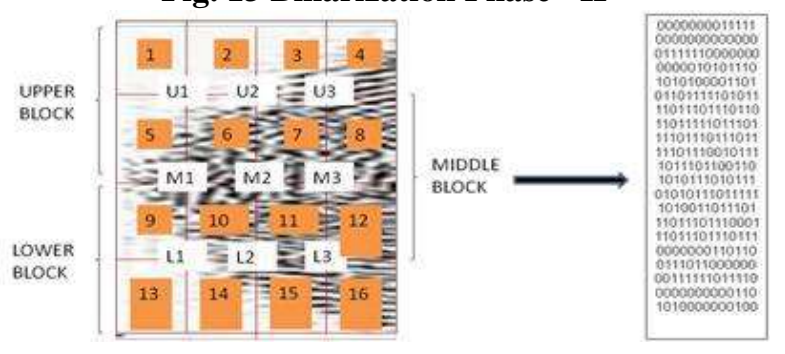

Fig. 14 Binarization Phase - III

\section{Feature extraction}

In this setting of feature extraction, we separate the picture into various settled estimated areas, every district has ON and OFF cells. 

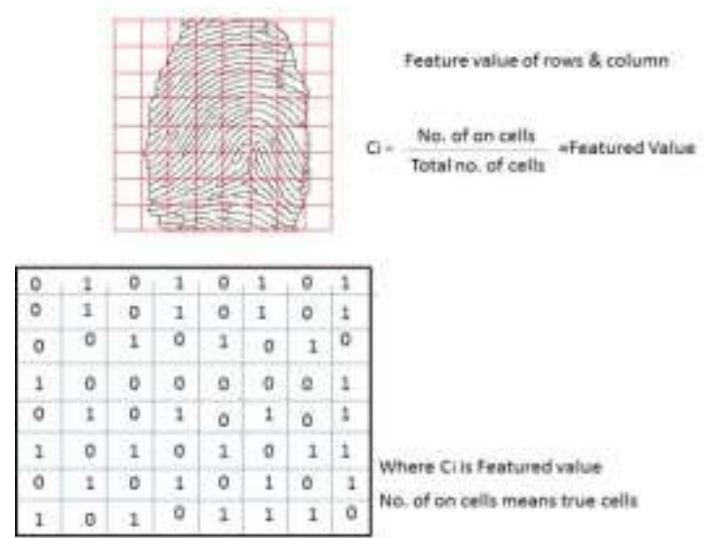

Fig. 15 Feature Extraction Process

We have the 19 regions which are considered are formed as a set of vectors shown below:

$\mathrm{V} 1$ : This is the leftmost part of the vertical regions.

$\mathrm{V} 2$ : This is the second part of the vertical regions.

$\square \quad$ V3 : This is the third part section of the vertical regions.

$\square \quad$ V4 : This is the fourth part section of the vertical regions.

$\square$ V5 : This is the rightmost part of the vertical regions.

$\square \mathrm{H} 1$ : This is the topmost part of the horizontal regions.

$\square \mathrm{H} 2$ : This is the second horizontal region from the top.

$\square \quad$ H3 : This is the third horizontal region from the top.

$\square \mathrm{H} 4$ : This is the fourth horizontal region from the top.

$\square \quad$ H5 : This is the bottom-most horizontal region.

$\square \quad \mathrm{U} 1$ : This is the set of $\{1,2,5,6\}$.

U2 : This is the set of $\{2,3,6,7\}$.

$\mathrm{U} 3$ : This is the set of $\{3,4,7,8\}$.

M1: This is the set of $\{5,6,9,10\}$.

M2: This is the set of $\{6,7,10,11\}$.

M3: This is the set of $\{7,8,11,12\}$.

L1 : This is the set of $\{9,10,13,14\}$.

L2 : This is the set of $\{10,11,14,15\}$.

L3 : This is the set of $\{11,12,15,16\}$.

So out of all these regions, M2 is considered as the central region. Feature values of all the regions are calculated. One of the examples is shown as below:

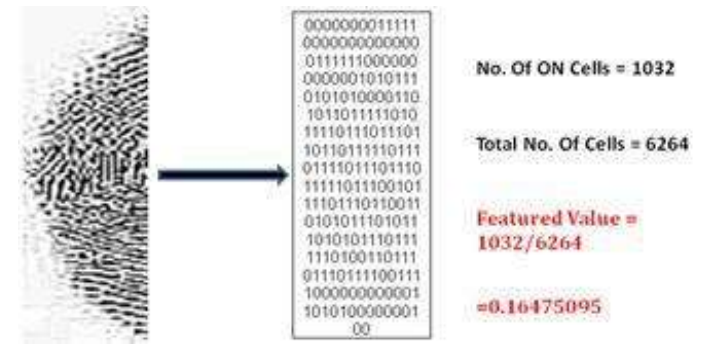

Fig. 16 Example of Feature Extraction For a sample
Feature matching

It includes all the stages from feature extraction from fingerprints to feature matching which generates a match score. The code is shown below for comparing the scores:

If (Featured Value $(\mathrm{Ci})>$ Threshold < Featured Value (Template) \&\& Featured Value (Ri) > Threshold < Featured Value (Template)) "Fingerprint is Matched" else "Fingerprint is not Matched"

\section{Implementation and Results}

Two lists are all around acknowledged to decide the execution of a unique finger impression acknowledgment framework: FRR (false rejection rate) and FAR (false acceptance rate).

The essential favourable position of our calculation is the precise outcome. Enlistment between two fingerprints is accomplished through amplification of common data, which is assessed from introduction field. This technique is substantially more like the human conduct when looking at two fingerprints. Accordingly, the blunders of misalignment, which regularly occurs in a details based enlistment, drastically diminished. False Rejection Rate (FRR): For a picture database, each example is coordinated against the rest of the specimens of a similar finger to register the False Acceptance Rate (FAR):

\begin{tabular}{|l|l|l|}
\hline Partition Value & FAR & FRR \\
\hline $1 \times 1$ & 0.19717 & 0.069 \\
\hline $2 \times 2$ & 0.08185 & 0.172 \\
\hline $3 \times 3$ & 0.04092 & 0.195 \\
\hline $4 \times 4$ & 0.00744 & 0.299 \\
\hline $5 \times 5$ & 0 & 0.35 \\
\hline
\end{tabular}

Table 1 Avg. FAR and FRR with Number of Partitions of an Image

The FAR and FRR both are conversely corresponding and relies on the nature of the picture whether the quality is great or bad. In expansion, the shared data gained in the enrollment arrange is consolidated into an official conclusion of coordinating outcome. In highlight coordinating, we propose a way to deal with the calculation of coordinating scores. The coordinating aftereffect of two component is given by a comparability level extending from 0 to 1 that demonstrates how precision they are coordinated, which gives a superior outcome than the basic "coordinated" or "unmatched". The above execution was a push to see how Unique finger impression Acknowledgment is utilized as a type of biometric to perceive characters of people. Different standard systems are utilized as a part of the middle phases of handling. We utilized a unique mark database from the FVC2004 for testing our analysis execution. Following outcomes depend on handling of 100 quantities of pictures: 


\begin{tabular}{|l|c|}
\hline Image DB & $\begin{array}{l}\text { Average Matching Time (in } \\
\text { Mili-Seconds) }\end{array}$ \\
\hline DB1 & 42 \\
\hline DB2 & 68 \\
\hline DB3 & 78 \\
\hline DB4 & 76 \\
\hline Avg. Time & 66 \\
\hline
\end{tabular}

Table 2 Evaluation of Matching Time

\begin{tabular}{|l|c|}
\hline Methods & $\begin{array}{l}\text { Avg. Matching Time (in Mili- } \\
\text { Seconds) }\end{array}$ \\
\hline $\begin{array}{l}\text { Mean and } \\
\text { Variance } \\
\text { Method[19] }\end{array}$ & 170 \\
\hline $\begin{array}{l}\text { Direction based } \\
\text { Method [20] }\end{array}$ & 120 \\
\hline $\begin{array}{l}\text { Gradient Based } \\
\text { Method [21] }\end{array}$ & 150 \\
\hline Proposed Method & 66 \\
\hline
\end{tabular}

\begin{tabular}{|l|l|l|l|l|}
\hline \multicolumn{1}{|c|}{ Table: 3 Comparison of Computational Time } \\
\hline $\begin{array}{l}\text { Quality } \\
\text { of } \\
\text { Image }\end{array}$ & $\begin{array}{l}\text { Match } \\
(\boldsymbol{\%}) \\
{[\mathbf{1 9}]}\end{array}$ & $\begin{array}{l}\text { Match } \\
(\boldsymbol{\%}) \\
{[\mathbf{2 0}]}\end{array}$ & $\begin{array}{l}\text { Match } \\
(\boldsymbol{\%}) \\
{[\mathbf{2 1}]}\end{array}$ & $\begin{array}{l}\text { Match } \\
(\%) \\
\text { Proposed } \\
\text { Method }\end{array}$ \\
\hline DB1 & 97 & 95 & 89 & 98 \\
\hline DB2 & 87 & 91 & 91 & 92 \\
\hline DB3 & 6 & 12 & 92 & 94 \\
\hline DB4 & 42 & 57 & 91 & 95 \\
\hline Average & 58 & 63.75 & 90.75 & 94.75 \\
\hline
\end{tabular}

Table 4: Average Accuracy Result

\begin{tabular}{|l|l|l|l|l|}
\hline $\begin{array}{l}\text { Size } \\
(\%)\end{array}$ & $\begin{array}{l}\text { Avg. } \\
\text { width } \\
\text { (Pixels) }\end{array}$ & $\begin{array}{l}\text { Avg. } \\
\text { Height } \\
\text { (Pixels) }\end{array}$ & FAR & FRR \\
\hline 100 & 200.00 & 300.00 & 0.0 & 0.350 \\
\hline 90 & 196.61 & 283.30 & 1.844871 & 1.090909 \\
\hline 80 & 185.32 & 267.07 & 1.988324 & 1.909091 \\
\hline 70 & 173.35 & 249.78 & 2.072143 & 2.181818 \\
\hline 60 & 160.42 & 231.22 & 2.675563 & 2.363636 \\
\hline 50 & 146.41 & 211.03 & 2.878649 & 3.636364 \\
\hline 40 & 130.90 & 188.70 & 2.905755 & 7.454545 \\
\hline 30 & 113.31 & 163.36 & 4.647206 & 13.09091 \\
\hline 20 & 92.42 & 133.30 & 9.611343 & 19.63636 \\
\hline 10 & 65.20 & 94.11 & 15.23937 & 45.63636 \\
\hline
\end{tabular}

Table 5: System Performances with Different Size of Same Images at $5 \times 5$ Partitions

\begin{tabular}{|l|l|l|}
\hline Method & FAR & FRR \\
\hline Spectrum Based [22] & $0.45 \%$ & $23.6 \%$ \\
\hline $\begin{array}{l}\text { (Spectrum + Direction) Based } \\
\text { [23] }\end{array}$ & $0.45 \%$ & $18.4 \%$ \\
\hline The Hybrid Fusion Based [24] & $0 \%$ & $6.6 \%$ \\
\hline $\begin{array}{l}\text { Partition Based (Proposed } \\
\text { Scheme) }\end{array}$ & $0 \%$ & $0.35 \%$ \\
\hline
\end{tabular}

Table 6: Comparison of FAR \& FRR of Four Schemes

Conclusion \& Future Enhancements
The aftereffects of a unique finger impression correlation are significantly something other than the subjective supposition of the analyst. In the lawful field, master witness declaration is displayed as sentiment declaration, not on account of the conclusion is somebody's closely-held conviction, but rather on the grounds that it is a conclusion that the layman is unequipped for framing. Unique mark recognizable proof frameworks are considered as being of high security quality. Since a fingerprint information is in the request of a few kilobytes, it gives the security of having a long watchword without the overhead of recalling that data. Be that as it may, not at all like a secret word framework, in which a correct match is normal for verification of an individual, a fingerprint acknowledgment framework can just give the person's character data with a specific confidence level. Along these lines, some sort of bending tolerant instrument is required which can diminish the security quality of the framework. In addition, with the modest number of highlights on a halfway fingerprint, the security quality of incomplete fingerprint acknowledgment is additionally decreased.

It is appropriate to conclude here with a brief summary of the future directions this work could take. The system could be used to perform matches on other images. For instance, character recognition could be performed. The only requirement is that the letters have enough contrast and possess a large enough spacing between minutiae. The system would perform extremely well with large printed images. This work has proven the design works. Now it can be implemented in multiprocessing environment to speed up processing time. The system could also be simulated on any suitable platform to allow portability across different operating systems. The last assignment is to apply the framework to a certifiable undertaking. The current coordinating calculation isn't exceptionally precise with low quality pictures; notwithstanding it could be incorporated with other acknowledgment frameworks, for example, confront acknowledgment. The subsequent mix would be an imposing security framework. Another work should be done in future is to handle the issue of seeking parameter space as the span of unique mark and the level of revolution increments. A decent streamlining calculation may address this issue. The majority of coordinating blunders are incited by the nonlinear distortion and low quality pictures. Because of the nearness of such a distortion, the flexible coordinating box must be sufficiently vast to endure it, which may build the likelihood of false coordinating.

\section{References}

1. Henry, E. R. (1900). Classification and Uses of Finger Prints.[Sl]: George Routledge and Sons.

2. Zhang, D. D. (2013). Automated biometrics: Technologies and systems (Vol. 7). Springer Science \& Business Media.

3. Jain, A. K., Ross, A., \& Prabhakar, S. (2004). An introduction to biometric recognition. IEEE 
Transactions on circuits and systems for video technology, 14(1), 4-20.

4. Maltoni, D., Maio, D., Jain, A. K., \& Prabhakar, S. (2003). Handbook of Fingerprint Recognition Springer. New York.

5. Jain, A. K., Nandakumar, K., \& Nagar, A. (2008). Biometric template security. EURASIP Journal on Advances in Signal Processing, 2008, 113.

6. Seow, B. C., Yeoh, S. K., Lai, S. L., \& Abu, N. A. (2002). Image based fingerprint verification. In Research and Development, 2002. SCOReD 2002. Student Conference on (pp. 58-61). IEEE.

7. Zhao, F., \& Tang, X. (2007). Preprocessing and postprocessing for skeleton-based fingerprint minutiae extraction. Pattern Recognition, 40(4), 1270-1281.

8. Moon, Y. S., Chen, J. S., Chan, K. C., So, K., \& Woo, K. C. (2005). Wavelet based fingerprint liveness detection. Electronics Letters, 41(20), 1112-1113.

9. Marana, A. N., \& Jain, A. K. (2005, October). Ridge based fingerprint matching using hough transform. In Computer Graphics and Image Processing, 2005. SIBGRAPI 2005. 18th Brazilian Symposium on (pp. 112-119). IEEE.

10. Lindoso, A., Entrena, L., Liu-Jimenez, J., \& San Millan, E. (2007). Correlation-based fingerprint matching with orientation field alignment. Advances in Biometrics, 713-721.

11. Ryu, C., Kim, H., \& Jain, A. K. (2006, August). Template adaptation based fingerprint verification. In Pattern Recognition, 2006. ICPR 2006. 18th International Conference on (Vol. 4, pp. 582-585). IEEE.

12. $\mathrm{Xu}$, H., Veldhuis, R. N., Kevenaar, T. A., \& Akkermans, T. A. (2009). A fast minutiae-based fingerprint recognition system. IEEE Systems journal, 3(4), 418-427.

13. Peralta, D., García, S., Benitez, J. M., \& Herrera, F. (2017). Minutiae-based fingerprint matching decomposition: Methodology for big data frameworks. Information Sciences, 408, 198-212.

14. Cappelli, R., Ferrara, M., \& Maltoni, D. (2012). Minutiae-based fingerprint matching. In Cross Disciplinary Biometric Systems (pp. 117-150). Springer Berlin Heidelberg.

15. Yuan, S., Zhang, G. Y., Wu, J. H., \& Guo, L. Y. (2014). Study of Fingerprint Image Preprocessing Technology. In Applied Mechanics and Materials (Vol. 519, pp. 581-584). Trans Tech Publications.

16. Hong, L., Wan, Y., \& Jain, A. (1998). Fingerprint image enhancement: Algorithm and performance evaluation. IEEE transactions on pattern analysis and machine intelligence, 20(8), 777-789.

17. Rajkumar, R., \& Hemachandran, K. (2012). A secondary fingerprint enhancement and minutiae extraction. Signal \& Image Processing, 3(2), 185.

18. Chen, W., Sui, L., Xu, Z., \& Lang, Y. (2012, May). Improved Zhang-Suen thinning algorithm in binary line drawing applications. In Systems and
Informatics (ICSAI), 2012 International Conference on (pp. 1947-1950). IEEE.

19. Peralta, D., Galar, M., Triguero, I., Miguel-Hurtado, O., Benitez, J. M., \& Herrera, F. (2014). Minutiae filtering to improve both efficacy and efficiency of fingerprint matching algorithms. Engineering Applications of Artificial Intelligence, 32, 37-53.

20. Yuan, C., Sun, X., \& Lv, R. (2016). Fingerprint liveness detection based on multi-scale LPQ and PCA. China Communications, 13(7), 60-65.

21. Xia, Z., Lv, R., Zhu, Y., Ji, P., Sun, H., \& Shi, Y. Q. (2017). Fingerprint liveness detection using gradientbased texture features. Signal, Image and Video Processing, 11(2), 381-388.

22. Galbally, J., Marcel, S., \& Fierrez, J. (2014). Image quality assessment for fake biometric detection: Application to iris, fingerprint, and face recognition. IEEE transactions on image processing, 23(2), 710724.

23. Helfroush, M. S., \& Mohammadpour, M. (2009, February). Fingerprint verification system: A nonminutiae based approach. In Computer, Control and Communication, 2009. IC4 2009. 2nd International Conference on (pp. 1-4). IEEE.

24. Yin, Y., Ning, Y., \& Yang, Z. (2010, September). A hybrid fusion method of fingerprint identification for high security applications. In Image Processing (ICIP), 2010 17th IEEE International Conference on (pp. 3101-3104). IEEE. 